\title{
Small Bowel Hemangioma in a 2-Year-Old Female With Recurrent Anemia
}

\author{
Jessica Coleman, MS, ${ }^{1}$ Ryan Phillips, MD, ${ }^{2}$ Rodney Steiner, MD ${ }^{1,2}$ \\ ${ }^{1}$ The University of Queensland School of Medicine, Ochsner Clinical School, New Orleans, LA ${ }^{2}$ Department of Pediatric Surgery, \\ Ochsner Clinic Foundation, New Orleans, LA
}

Background: Hemangiomas of the small bowel are rare tumors that present with melena, hematochezia, or signs of anemia. Because of the rarity of gastrointestinal hemangiomas, they are not commonly considered in the differential diagnosis for pediatric patients with anemia.

Case Report: We present a case of small bowel hemangioma in a 2-year-old female with recurrent episodes of severe anemia. After resection of a $4.5 \mathrm{~cm} \times 2 \mathrm{~cm}$ benign hemangioma, the patient's hemoglobin level steadily improved. The patient did not experience recurrence and has had no problems with activities of daily living.

Conclusion: Small bowel hemangiomas can cause serious life-threatening anemia or obstruction of the small bowel and should be considered in cases of pediatric anemia unresponsive to medical management.

Keywords: Gastrointestinal hemorrhage, intestine-small, pediatrics, vascular neoplasms

Address correspondence to Rodney Steiner, MD, Department of Pediatric Surgery, Ochsner Clinic Foundation, 1514 Jefferson Hwy., New Orleans, LA 70121. Tel: (504) 842-3907. Email: rodney.steiner@ochsner.org

\section{INTRODUCTION}

Gastrointestinal (Gl) hemangiomas are rare, benign vascular tumors. They are especially rare in the pediatric population. Gl hemangiomas can occur throughout the Gl tract but occur most commonly in the small bowel, colon, and rectum. ${ }^{1}$ Symptoms of $\mathrm{Gl}$ hemangiomas are the signs of Gl bleeding: melena, hematochezia, and anemia. Symptoms can be severe, and patients often require multiple blood transfusions. Because of the rarity of GI hemangiomas, they are not often considered as a cause of anemia or Gl bleeding in children. We present another case of small bowel hemangioma in a pediatric patient.

\section{CASE REPORT}

A 2-year-old female born at 39 weeks, 4 days presented with severe anemia. She had a significant medical history of severe microcytic anemia requiring multiple admissions and transfusions. When she was 23 months of age (prior to her first hospital admission), the patient was diagnosed with incidental anemia; her hemoglobin was $3.8 \mathrm{~g} / \mathrm{dL}$. Workup at the time of her first hospital admission resulted in a diagnosis of severe iron deficiency anemia secondary to poor intake. Prior to this first admission, the patient had been treated with supplemental iron and strict dairy restriction as recommended by her pediatrician for her symptoms but had shown no improvement. However, some noncompliance with the treatment of supplemental iron and dairy restriction was suspected. At the first admission, the patient was transfused with $10 \mathrm{~mL} / \mathrm{kg}$ of packed red blood cells (RBCs) and had a hemoglobin of $6.3 \mathrm{~g} / \mathrm{dL}$ on discharge after 1 day of hospital stay with instructions to follow up with hematology/oncology the following week. At follow-up with hematology/oncology, she was much improved symptomatically and had a hemoglobin of $6.5 \mathrm{~g} / \mathrm{dL}$. The parents were instructed to continue administering the supplemental iron and limiting milk consumption.

Two months later, the patient presented to the emergency department with pallor, and her laboratory workup showed a hemoglobin level of $2.8 \mathrm{~g} / \mathrm{dL}$. She received two transfusions of $5 \mathrm{~mL} / \mathrm{kg}$ packed RBCs and one transfusion of $10 \mathrm{~mL} / \mathrm{kg}$ packed RBCs and was discharged after 2 days.

Follow-up appointments with pediatric gastroenterologists, scheduled after the hospital admission when the patient had the hemoglobin level of $2.8 \mathrm{~g} / \mathrm{dL}$, were to include a Meckel workup, colonoscopy, and esophagogastroduodenoscopy, but laboratory workup prior to her gastroenterology appointment (2.5 months after her first hospital admission) indicated a hemoglobin of $3.3 \mathrm{~g} / \mathrm{dL}$ and reticulocyte count of $17 \%$, resulting in another admission.

The parents denied any melena or hematochezia but stated that the patient's stools were often "dark and almost black." The patient's stool was positive for occult blood. After 3 transfusions of $10 \mathrm{~mL} / \mathrm{kg}$ packed RBCs, the patient's hemoglobin improved to $10 \mathrm{~g} / \mathrm{dL}$. Meckel scan performed during admission was initially read as negative, but an addendum noted simultaneous accumulation of radiotracer 


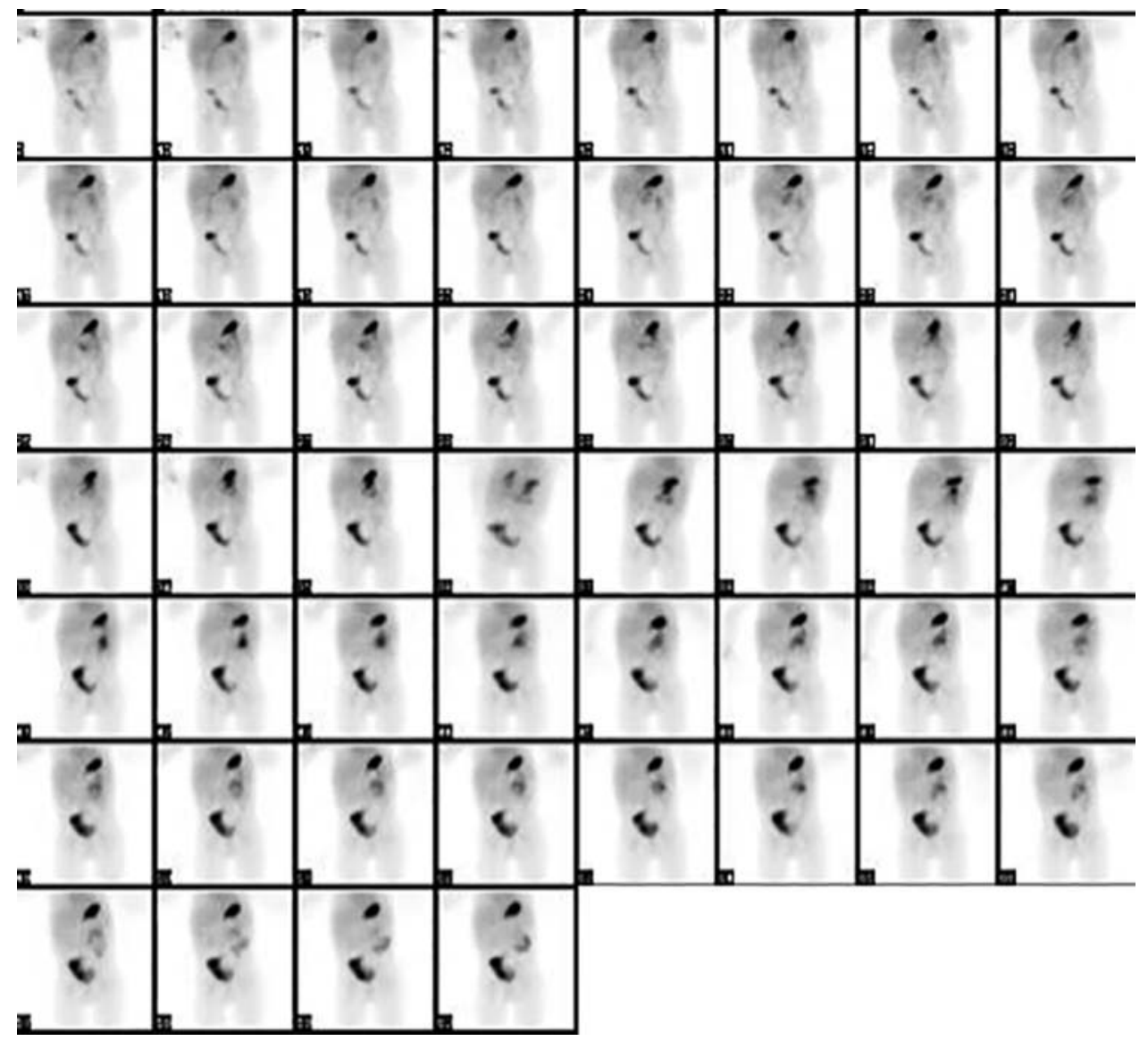

Figure 1. Meckel scan in a patient with recurring anemia showed transit of excreted radiotracer throughout the small bowel and proximal colon that demonstrates peristalsis. This finding is favored to be artifactual within the right colon without definite evidence of focal uptake in the region of the expected location of a Meckel diverticulum. An addendum report identified apparent simultaneous accumulation of radiotracer in the right lower quadrant in the stomach suspicious for a Meckel diverticulum.

in the right lower quadrant of the stomach suspicious for a Meckel diverticulum (Figure 1). Physical examination was significant for extreme pallor.

The patient was taken to the operating room on day 3 of the current admission for repair of a presumed Meckel diverticulum. During surgery, a vascular malformation of the small bowel measuring $4.5 \mathrm{~cm} \times 2 \mathrm{~cm}$ was found on the antimesenteric surface. The lesion was not actively bleeding but invaded the wall of the small bowel by approximately $6 \mathrm{~cm}$ (Figure 2). The lesion was resected, and pathology reported the specimen to be a benign hemangioma of the small bowel with focal fibrosis, calcification, and thrombosis (Figure 3 ).

The patient was discharged after 5 days (postoperative day 2). Two weeks after surgery, the patient's hemoglobin level was $6.8 \mathrm{~g} / \mathrm{dL}$. At 3-month follow-up, the patient had a hemoglobin level of $10 \mathrm{~g} / \mathrm{dL}$. The patient did not experience recurrence of anemia and has had no problems with activities of daily living.

\section{DISCUSSION}

Hemangiomas in the small intestine are rare benign tumors and account for approximately $5 \%$ of all GI neoplasms. ${ }^{2}$ GI hemangiomas typically occur in the ileum and are rarely found in children. A literature review revealed only 9 known cases (Table).$^{2-8}$ Patients with GI hemangioma present with melena and hematochezia, sometimes causing severe anemia, or with symptoms of obstruction. Patients may also exhibit abdominal discomfort, abdominal pain, dizziness, nausea, vomiting, fatigue, general weakness, and malaise. As noted earlier, GI hemangiomas may present in the small bowel, the colon, and the rectum, with the small bowel being the most frequent site. ${ }^{1} \mathrm{Gl}$ hemangiomas may be a manifestation of Maffucci syndrome, Klippel-Trenaunay syndrome, disseminated neonatal hemangiomatosis, or blue rubber bleb nevus syndrome. ${ }^{1}$ Gl hemangiomas are classified histologically as capillary, cavernous, and mixed types. ${ }^{3}$ Capillary hemangiomas, also referred to as pyogenic granulomas, are usually solitary and induce anemia by chronic bleeding, whereas cavernous hemangiomas induce hematemesis and melena from acute hemorrhaging. ${ }^{1}$ Cavernous hemangiomas occur more frequently than capillary hemangiomas.

Fawcett et al reported the cases of 2 pediatric patients with GI tract abnormalities who were discovered to have immunologic defects in both humoral and cellular immunity. ${ }^{9}$ Both patients presented with an array of immunologic 


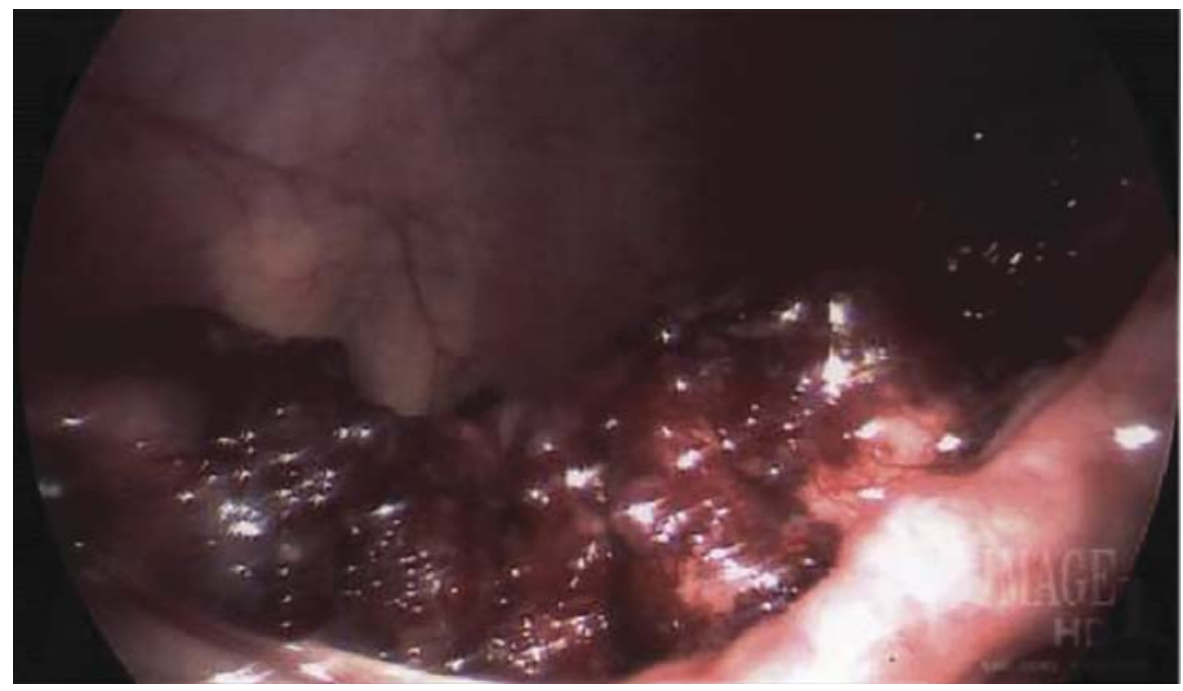

Figure 2. Operative findings indicated a vascular malformation.

deficits and anemia. After surgical correction of the Gl abnormalities, all immunologic values improved. By 10-18 months after surgery, both children had normal immune function. These findings suggest that lesions of the Gl tract could be masquerading as common childhood disorders by causing secondary immunodeficiency. ${ }^{9}$

Diagnosis of GI hemangioma relies on computed tomography, magnetic resonance imaging, scintigraphy, angiography, and abdominal ultrasonography. These tools are accurate regardless of patient age or presentation. Depending on the location and features of the lesion, esophagogastroduodenoscopy or colonoscopy can be used to isolate a GI hemangioma. ${ }^{10}$ Capsule endoscopy has been found to be useful in diagnosis as well but can be difficult for patients who are too young to swallow large pills and is contraindi- cated in patients with potential obstructions. ${ }^{7}$ Wireless capsule endoscopy is a first-line modality for evaluation of the small bowel in adults but has not been determined to be first line in the pediatric population yet. ${ }^{7} \mathrm{~A}$ case of a small bowel hemangioma in a 2.5-year-old was successfully diagnosed with wireless capsule endoscopy in 2006. ${ }^{7}$ Ge et al studied wireless capsule endoscopy in 2007 and determined it to be effective and safe in diagnosing children with Gl bleeding and comparable to diagnosis in adults. ${ }^{11}$ A technique for assessing small bowel disorders developed in 2011 is double-balloon enteroscopy (DBE). Lin and Erdman investigated DBE in the pediatric population and determined that it is safe for diagnosis and treatment, but they noted that DBE should be reserved for patients with a high suspicion for small bowel pathology after other less-invasive

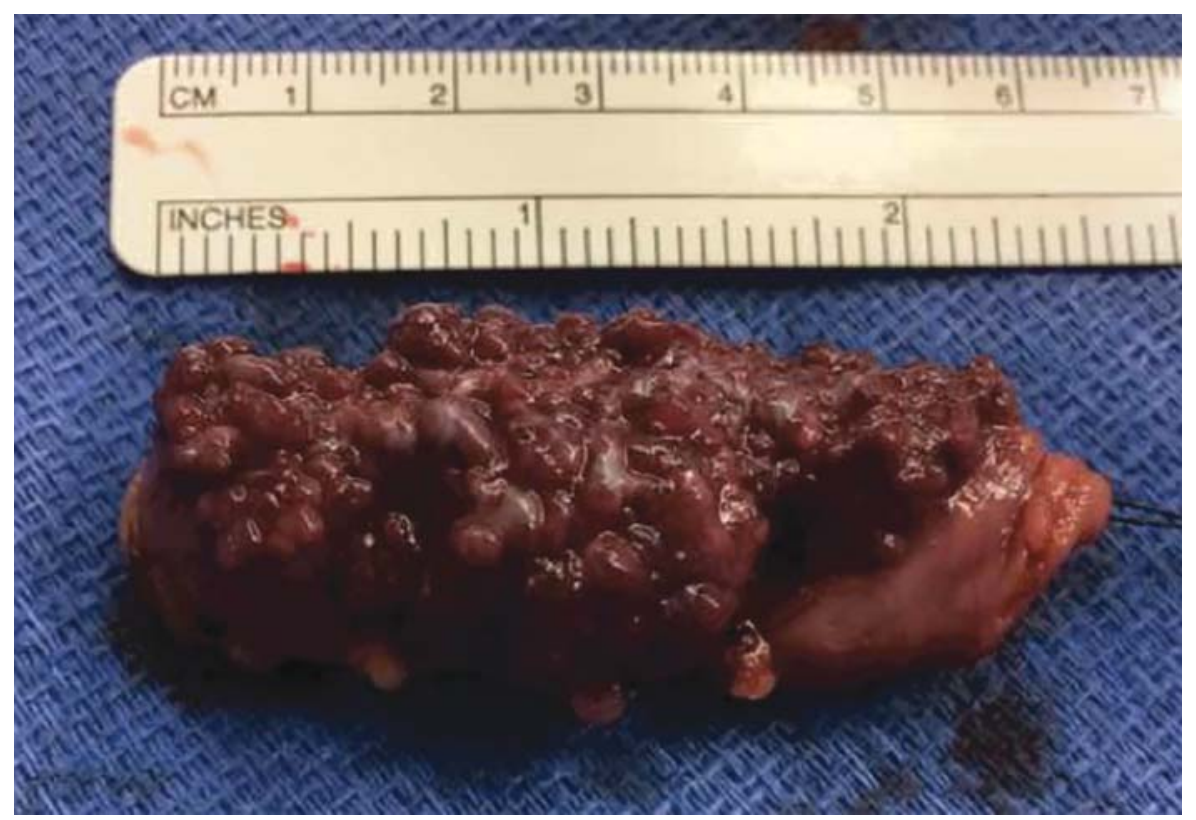

Figure 3. Pathology determined that the $4.5 \mathrm{~cm} \times 2 \mathrm{~cm}$ specimen was a benign hemangioma of the small bowel with focal fibrosis, calcification, and thrombosis. 
Coleman, J

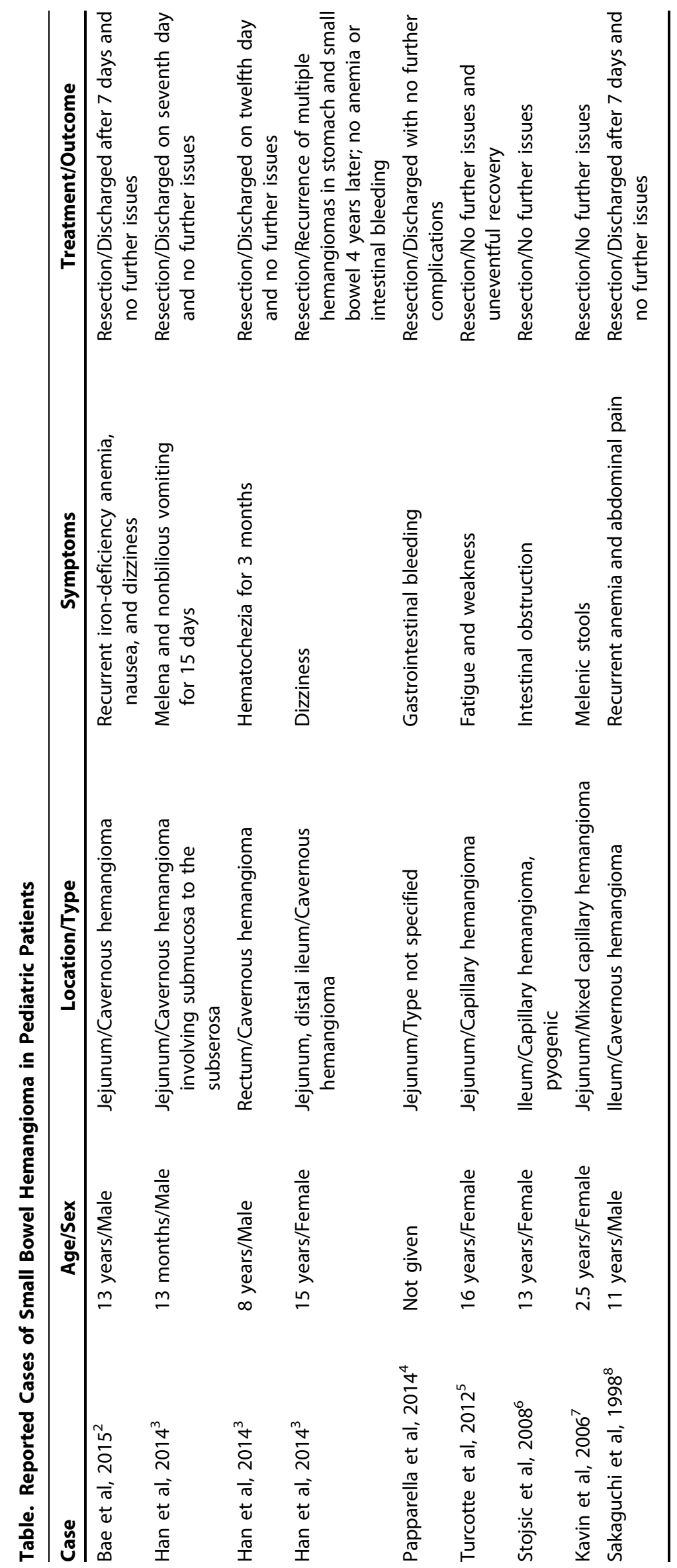


techniques have failed to diagnose the condition. ${ }^{12}$ The diameter of the enteroscope is $12.2 \mathrm{~mm}$, so it has the potential to cause abdominal pain, Gl tract tears, and acute pancreatitis. ${ }^{13}$ Additionally, a study of Meckel diverticulum interestingly discovered a patient with a small bowel hemangioma of the jejunum who also had a positive Tc99 scan, suggesting a potential diagnostic role. ${ }^{4}$

Treatment of GI hemangiomas includes addressing symptoms, observation, and follow-up. Iron supplements and transfusions of packed RBCs should be used in patients with anemia. Corticosteroids, which suppress the expression of vascular endothelial growth factor-A, can also be used. ${ }^{14}$ Surgical intervention should be considered for patients with symptoms that do not respond to medical management. $\mathrm{Pa}-$ tients with comorbidities, large lesions, multiple lesions, or severe GI bleeding can benefit from surgical intervention. Hemangiomas in the small bowel should be treated with segmental resection, whereas colon lesions should be treated with ileocecectomy, right hemicolectomy, left hemicolectomy, or segmental resection, depending on the location and extent of the hemangioma. ${ }^{3}$

\section{CONCLUSION}

Although the incidence is rare, small bowel hemangioma can cause serious life-threatening symptoms and should be considered in cases of pediatric anemia unresponsive to medical management.

\section{ACKNOWLEDGMENTS}

The authors have no financial or proprietary interest in the subject matter of this article. The authors would like to acknowledge the patient and the patient's guardians for being willing to publish her case as well as the team that provided care for the patient.

\section{REFERENCES}

1. Magnano A, Privitera A, Calogero G, Nanfito' L, Basile G, Sanfilippo G. Solitary hemangioma of the small intestine: an unusual cause of bleeding diagnosed at capsule endoscopy. $J$ Pediatr Surg. 2005 Oct;40(10):e25-e27.

2. Bae SJ, Hwang G, Kang HS, et al. Single cavernous hemangioma of the small bowel diagnosed by using capsule endoscopy in a child with chronic iron-deficiency anemia. Clin Endosc. 2015 Jul;48(4):340-344. doi: 10.5946/ce.2015.48.4.340.
3. Han EC, Kim SH, Kim HY, Jung SE, Park KW. Gastrointestinal hemangioma in childhood: a rare cause of gastrointestinal bleeding. Korean J Pediatr. 2014 May;57(5):245-249. doi: 10.3345/kjp.2014.57.5.245.

4. Papparella A, Nino F, Noviello C, et al. Laparoscopic approach to Meckel's diverticulum. World J Gastroenterol. 2014 Jul 7;20 (25):8173-8178. doi: 10.3748/wjg.v20.i25.8173.

5. Turcotte JF, Prasil P, Gagnon P, Castilloux J. Case 1: recurrent iron-deficiency anemia in a teenager. Paediatr Child Health. 2012 Jan;17(1):21-22.

6. Stojsic Z, Brasanac D, Kokai G, et al. Intestinal intussusception due to a pyogenic granuloma. Turk J Pediatr. 2008 Nov-Dec;50 (6):600-603.

7. Kavin H, Berman J, Martin TL, Feldman A, Forsey-Koukol K. Successful wireless capsule endoscopy for a 2.5-year-old child: obscure gastrointestinal bleeding from mixed, juvenile, capillary hemangioma-angiomatosis of the jejunum. Pediatrics. 2006 Feb;117(2):539-543.

8. Sakaguchi M, Sue K, Etoh G, et al. A case of solitary cavernous hemangioma of the small intestine with recurrent clinical anemic attacks in childhood. J Pediatr Gastroenterol Nutr. 1998 Sep;27(3):342-343.

9. Fawcett WA 4th, Ferry GD, Gorin LJ, Rosenblatt HM, Brown BS, Shearer WT. Immunodeficiency secondary to structural intestinal defects. Malrotation of the small bowel and cavernous hemangioma of the jejunum. Am J Dis Child. 1986 Feb;140(2):169-172.

10. Park JH. Role of colonoscopy in the diagnosis and treatment of pediatric lower gastrointestinal disorders. Korean J Pediatr. 2010 Sep;53(9):824-829. doi: 10.3345/kjp.2010.53.9.824.

11. Ge ZZ, Chen HY, Gao YJ, Gu JL, Hu YB, Xiao SD. Clinical application of wireless capsule endoscopy in pediatric patients for suspected small bowel diseases. Eur J Pediatr. 2007 Aug;166 (8):825-829. doi: 10.1007/s00431-006-0331-9.

12. Lin TK, Erdman SH. Double-balloon enteroscopy: pediatric experience. J Pediatr Gastroenterol Nutr. 2010 Oct;51(4):429432. doi: 10.1097/MPG.0b013e3181d2979c.

13. Liu W, Xu C, Zhong J. The diagnostic value of double-balloon enteroscopy in children with small bowel disease: report of 31 cases. Can J Gastroenterol. 2009 Sep;23(9):635-638.

14. Cho JS, Kang JH, Park IH, Lee HM. Steroids inhibit vascular endothelial growth factor expression via TLR4/Akt/NF-kB pathway in chronic rhinosinusitis with nasal polyp. Exp Biol Med (Maywood). 2014 Aug;239(8):913-921.

This article meets the Accreditation Council for Graduate Medical Education and the American Board of Medical Specialties Maintenance of Certification competencies for Patient Care and Medical Knowledge. 